\title{
Higher-Periodic and Aperiodic Stick-Slip Dynamics in a Friction Damper
}

\author{
Naresh K. Chandiramani \\ Department of Civil Engineering, Indian Institute of Technology, Bombay 400076, India
}

(Received 9 January 2005; accepted 11 January 2006)

\begin{abstract}
A single-degree-of-freedom friction wedge damper suspension model is considered with nonlinear stiffness and viscous damping included. The response to harmonic excitations is obtained for the Coulomb, slip-rate, and smooth friction formulations. Since the convergence towards steady state is slow, especially for higher excitation frequencies, a modified shooting method is used when seeking periodic responses for the discontinuous friction models. The concept of stationary flow curves is used for stick-slip analysis when considering the discontinuous friction models. The coexistence of multiple stick regions - which could further complicate the dynamics - is obtained as a direct and nontrivial consequence of the stiffness nonlinearity. Results depicting stick displacements versus forcing amplitude, forcing frequency, wedge angle, and sideframe angle, are reported. The stick displacements are higher when a softening stiffness nonlinearity is present. Period multiplications, demultiplications, and chaotic responses are obtained when nonlinear stiffness (cubic) and viscous damping are considered and the track excitation frequency is varied. The present analysis, with nonlinearities included, could serve as a more realistic plant model in compensator design for real-time control.
\end{abstract}

\section{Nomenclature}

$\mu_{k}, \mu_{s}-$ dynamic and static friction coefficients, respectively, in Coulomb and smooth models

$\mu_{1}, m_{1}, m_{2}, m_{3}-$ coefficients in slip-rate model

$\eta_{1}, \eta_{2}-$ coefficients in the smooth model

$\alpha ; \gamma \quad$ - wedge angle; sideframe angle

$\mu_{w s}, \mu_{w b}$ - friction coefficients at wedge-sideframe and wedge-bolster interface, respectively

$Y_{w o}, Y_{b o}-$ static preloading in wedge and bolster springs, respectively

$Y_{w}, Y_{b}$ - vertical displacement of wedge and bolster, respectively, relative to sideframe

$Y_{s f} ; A_{T} ; \Omega ; t$ - vertical motion of sideframe; track amplitude; track frequency; time

$X_{w} \quad$ - horizontal displacement of wedge relative to sideframe centreline

$y ; \beta ; a ; \tau$ - nondimensional-bolster displacement; track frequency; track amplitude; time

$y_{1}, y_{2}-$ components of state vector $\boldsymbol{y}$, i.e., non-dimensional bolster displacement and velocity

$y_{s k}^{ \pm}, y_{s k 1}^{ \pm}, y_{s k 2}^{ \pm}, y_{s k 3}^{ \pm}-$stationary flow solutions of $y_{1}$ justabove $(+)$ and below $(-)$ the stick plane

$y_{1 \text { entry }}, \tau_{\text {entry }}-$ values of $y_{1}$ and $\tau$, respectively, when trajectory enters the stick plane

$y_{\text {stick }} ; \tau_{\text {exit }}-$ stick displacement; value of $\tau$ when trajectory exits the stick region

$\eta_{0} ; \delta \eta$ - starting guess of initial condition $(\eta)$ of state lying on the periodic trajectory being sought; and its correction as obtained via shooting

$T_{0}, j$ - response period (integral multiple of track excitation period); period number

$g \quad$ - gravitational acceleration

$\nabla_{\eta} \boldsymbol{y}$ - Jacobian matrix of state relative to initial condition, having components $y_{3}, y_{4}, y_{5}, y_{6}$.

$\boldsymbol{f} ; \nabla_{\boldsymbol{y}} \boldsymbol{f}$ - forcing vector in IVP Eq. (3); and its Jacobian matrix relative to state vector
$M_{b} ; M_{w}$ - bolster mass (including car); wedge mass

$K_{b}, K_{b 2}, K_{b 3} ; K_{w}, K_{w 2}, K_{w 3}$ - linear, quadratic, cubic stiffness coefficients, respectively, for bolster and wedge spring nests, respectively

$\omega-$ natural frequency of bolster spring-mass system, defined in Eq. (A1)

$c_{w}, c_{w_{3}} ; c_{b}, c_{b_{3}}-$ linear, cubic viscous damping coefficients of wedge and bolster dampers

$p_{2}, p_{3} ; c_{1}, c_{3}$ - quadratic, cubic stiffness ratios; and linear, cubic viscous damping ratios, defined in Eq. (A1)

$C_{l}^{ \pm}, C_{c}^{ \pm} ; F^{ \pm}, J_{q}^{ \pm}, J_{c}^{ \pm} ; H^{ \pm} ; G^{ \pm}$- nondimensional coefficients of linear and cubic viscous damping; linear, quadratic, and cubic stiffness; preloading; track excitation, above $(+)$ and below $(-)$ the stick plane, defined in terms of suspension parameters, Eq. (A1)

\section{INTRODUCTION}

Frictional damping finds application in turbine blades, robotics, etc. In railroad vehicles, such as the three-piece freight truck, friction wedge devices are commonly used as damping elements in the secondary suspension. ${ }^{1}$ Due to frictional contact and nonlinear spring stiffness and viscous damping, these devices can yield complicated dynamics which may include aperiodic and stick-slip motions, the latter appearing when discontinuous friction laws are used. Moreover, fast prediction of these responses is essential when combining real-time active vibration control with passive energy dissipation via friction and viscous elements.

Periodic stick-slip motion was obtained by Den Hartog for a single-degree-of-freedom system with dry friction arising from constant normal load. ${ }^{2}$ Anderson and Ferri considered dry friction with displacement and velocity dependent normal force and showed that, for certain forcing frequencies, increasing the displacement-dependent friction could (surprisingly) raise the response amplitude. ${ }^{3}$ Narayanan and Jayaraman considered a system with dry friction, self and external 